\title{
SPATIAL-SPECTRAL MORPHOLOGICAL FEATURE EXTRACTION FOR HYPERSPECTRAL IMAGES CLASSIFICATION
}

\author{
M. Dowlatshah ${ }^{1}$, H. Ghassemian ${ }^{1, *}$, M. Imani ${ }^{1}$ \\ ${ }^{1}$ Image Processing and Information Analysis Laboratory, Faculty of Electrical and Computer Engineering, \\ Tarbiat Modares University, Tehran, Iran \\ m.dowlatshah@modares.ac.ir, ghassemi@modares.ac.ir,maryam.imani@modares.ac.ir
}

\section{Commission VI, WG VI/4}

KEY WORDS: Hyperspectral, Feature Extraction, Spatial Features, Spectral Features, Morphology Profiles

\begin{abstract}
:
Remote sensing image classification is a method for labeling pixels to show the Land cover types. The ambiguity in the classification process can be reduced if the spatial dependencies, which exist among the adjacent pixels, are intelligently incorporated into the feature extraction process. One of the methods for spatial feature extraction is applying morphological filters. The basic idea of the morphological filters is comparison of structures within the image with a reference form called structural element. Four types of important morphological filters are included (dilation, erosion, opening, and closing) in this work. Opening morphological filter is used to extract spatial features where this filter is implemented by applying two successive sequences dilation and erosion operators. This filter removes the light areas smaller than the structural element in binary images; and in the gray level images, the areas smaller than the structural element and brighter than the neighboring regions are removed. Differential morphology filters are other important morphological filters, which are also used in this work. In the proposed method, the principal component analysis is used to reduce the data dimensions and an SVM classifier is applied to classify the hyperspectral data. The proposed method provides better classification results than the conventional morphological profile about 2\%-5\% for the University of Pavia and Pavia Center datasets. The results represent the good performance of the proposed method by using a small number of training samples.
\end{abstract}

\section{INTRODUCTION}

In the classification of remote sensing images, the aim is to determine the class of each region in the image. The ambiguity in the classification process can be reduced if the spatial dependencies, which exist among the adjacent pixels, are intelligently incorporated into the decision making process. There are generally 2 types of information available, spatial information and spectral information (Ghassemian and Landgrebe, 1988a). The images received from the sensors are in the form of a 3-dimensional matrix with spatial information in the matrix rows and columns and spectral information at the depth of the matrix. Each vector in the row and column of this matrix is a pixel of the image (Imani and Ghassemian, 2016a). In a spectral-based classification, pixels will not change randomly the function of the classifier, if the spatial properties in the images give us more information than the spectral in the image, and improve the performance of the classifier. The proposed method uses spectral and spatial information to classify data simultaneously (Golipour et al. 2016; Khodadadzadeh and Ghassemian, 2011).

The process of identifying, detecting complications and objects on the scene, using the images obtained by the sensors, is called remote sensing. The use of aerial and spatial images to interpret, identify, and obtain information from phenomena in surveying and studying land resources is called remote sensing. The use of aerial images and space images for the interpretation, identification, and acquisition of information from phenomena in the study of ground resources, is called remote sensing (Pesaresi and Benediktsson, 2001).
With the improvement of remote sensing techniques, the hyperspectral images contain high spectral and spatial resolutions and so provide new possibilities and challenges for land-cover classification. The spatial-spectral analysis of hyperspectral images is based on spectral bands measurements and a series of features extracted from neighbouring pixels using spatial operations (Mirzapour and Ghassemian, 2016a), (Mirzapour and Ghassemian, 2016b), (Kianisarkalehn and Ghassemian, 2016). The morphological profile (MP) attempts to model spatial information by analyzing the interaction of a set of structural elements with different shapes and sizes. The MP based on opening and closing by reconstruction (Benediktsson et al. 2003), differential morphological profile (DMP) (Dalla Mura et al. 2010a), extended morphological profile (EMP), attribute profile, and extended attribute profile (Dalla Mura et al. 2010b) have been introduced to include spatial (shape and size) information into classification result (Imani and Ghassemian, 2016c).

So far, several methods have been used to analysis the spatial features of remote sensing images (Ghassemian and Landgrebe, 1988b; Chaji and Ghassemian, 2006). One of the important methods is the use of morphological filters, which include four important filters: dilation, erosion, opening, and closing. Opening and closing filters are obtained using dilation and erosion filters, which are described in more details in the following sections (Roochi and Ghassemian, 2017). Morphological operations are nonlinear operations based on the theory of collections and processing binary images. By expanding the concepts of these operations, they are used to process non-binary images, namely gray-level images and then to process multi-band images. Morphological filters are one of

\footnotetext{
* Corresponding author
} 
the spatial feature extraction methods in which differential morphological filters are formed based on them. By using differential morphological filters, we can extract a series of important edges and structural information from the image, which improves the output of the classifier. To use the concept of morphological profile in the processing of hyperspectral images, with the development of such profiles, the extended morphology profile was introduced. In fact, the procedure of this profile is based on extracting morphological profiles from a set of images extracted from hyperspectral data. The use of these profiles along with spectral features also has positive results for the classification of hyperspectral data (Fauvel et al. 2013). After the success of morphological filters and their differentiation in the extraction of spatial features of remote sensing data, another set of nonlinear filters based on morphological operations, called attribute filters, are used to extract spatial data from single-band and multi-band data. In this regard, various techniques have been used for the extraction and classification of remote sensing images, which have been studied for the proposed method.

The order of the various sections of the article is as follows: In section 2, MP, EMP, DMP, and the proposed method are explained. In section 3 , we show the results of the experiments using two hyperspectral data. Finally, conclusions are provided in Section 4.

\section{MORPHOLOGICAL PROFILE FOR HYPERSPECTRAL DATA}

In this section, we explain morphological filters, extended morphological filters, and differential morphological filters. Morphological filters are a set of nonlinear operations designed to compare structures inside the image with a reference form called structural element. Extended morphological filters are obtained using morphological filters that are used for highdimensional data and differential morphological filters are used for extracting information such as edge and target structures (Imani and Ghassemian, 2017).

Erosion: By applying this operator to a grayscale image, the minimum gray levels associated with the pixels of the image below the structural element are selected as the corresponding value in the output image. In this way, areas of the image that are brighter than the surrounding areas will erode.

Dilation: By applying the dilation operator to binary images, if all the image pixels located below the structural element are zero, the corresponding result in the output image is zero and otherwise it will be one. Similarly, in applying this operator to a gray level image, the maximum gray levels associated with the image pixels located below the structural element are selected as the corresponding value in the output image (Roochi and Ghassemian, 2017).

\subsection{Mathematics Morphological}

Mathematics morphological collections represent the objects in an image. For example, a set of all white pixels in an image is a morphological description of the image. In the binary images of the sets concerned, the members of the set of integers are twodimensional, in which each member of a set is a set of whose coordinates are $(\mathrm{x}, \mathrm{y})$ of a white pixel (or black, depending on the arrangement) in the image. Grayscale digital images can be described with sets whose members are $z^{2}$. In this case, two parts of each set member refer to the pixel coordinates, and the third represents the discrete amount of intensity. Collections in higher-dimensional spaces can display other features of the image, such as color and variable-time components. Reconstruction is a morphological transformation that includes two input images: an image called a marker and an image called a mask. For binary images, the marker must be a sub-mask. For gray level images, the values of gray surfaces must be less than or equal to the corresponding values in the mask. Usually, mask is the main image and the marker is made from the mask. In the following equations, the opening and closing filters are shown as $\gamma_{R}$ and $\varphi_{R}$, respectively.

$\gamma_{R}^{i}(f)=R_{f}^{\varepsilon}\left(\varepsilon^{i}(f)\right)$

$\varphi_{R}^{i}(f)=R_{f}^{\delta}\left(\delta^{i}(f)\right)$

Where, $\varepsilon^{i}$ and $\delta^{i}$ respectively represent the erosion and dilation filters, I is the size of the structural element, and $R_{f}^{\delta}$ $R_{f}^{\varepsilon}$ respectively, morphological filters, are the dilation and erosion of reconstruction. A morphology profile is obtained by using morphological filters of opening and closing. Opening and closing morphological profiles displayed respectively, with $\Pi_{\gamma}$ and $\Pi_{\varphi}$, contain a series of opening and closing filters.

The formula for a morphological profile is as follows:

$$
\begin{aligned}
& M P(f)=\Pi_{i}\left\{\Pi_{i}=\Pi_{\varphi \lambda}, \lambda=(n-1+i), \forall \lambda \in[1, n]\right\} \\
& M P(f)=\Pi_{i}\left\{\Pi_{i}=\Pi_{\varphi \lambda}, \lambda=(i-n-1), \forall \lambda \in[n+1,2 n+1]\right\}
\end{aligned}
$$

The opening morphological filters remove areas that are smaller than the structural element; also the closing morphological filters remove same areas. The difference is that opening filters remove areas that are brighter than neighboring regions, but closing filters eliminate areas that are darker than the neighboring regions.

\subsection{Extended Morphological Profiles}

Extended morphological profiles are derived using morphological profiles. With the expansion of concepts and data, the use of extended morphology profiles has become widespread. These types of filters are used for high-dimensional data. To avoid the complexity of the calculations, the principal component analysis method is used to reduce the dimension of the data. Principal Component Analysis (PCA) is a transformation in vector space, which is further used to reduce the size of the data set. With this transformation, the dimensions become unevenly matched and the covariance matrix of the data becomes diagonal (Plaza et al. 2005). This transformation creates a space in which the data are arranged according to the amount of the variance; the data with the most variance is transferred to the first dimension, and the rest of the data are transferred to next dimensions according to their variance amount. Therefore the last main component has the least variance. This can be interpreted as the later ones have a small share in the resolution and can be ignored (Fauvel et al. 2007). The EMP formula using the principal component analysis is as follows:

$$
E M P=\left\{M P\left(P C_{1}\right), M P\left(P C_{2}\right), \ldots, M P\left(P C_{n}\right)\right\}
$$




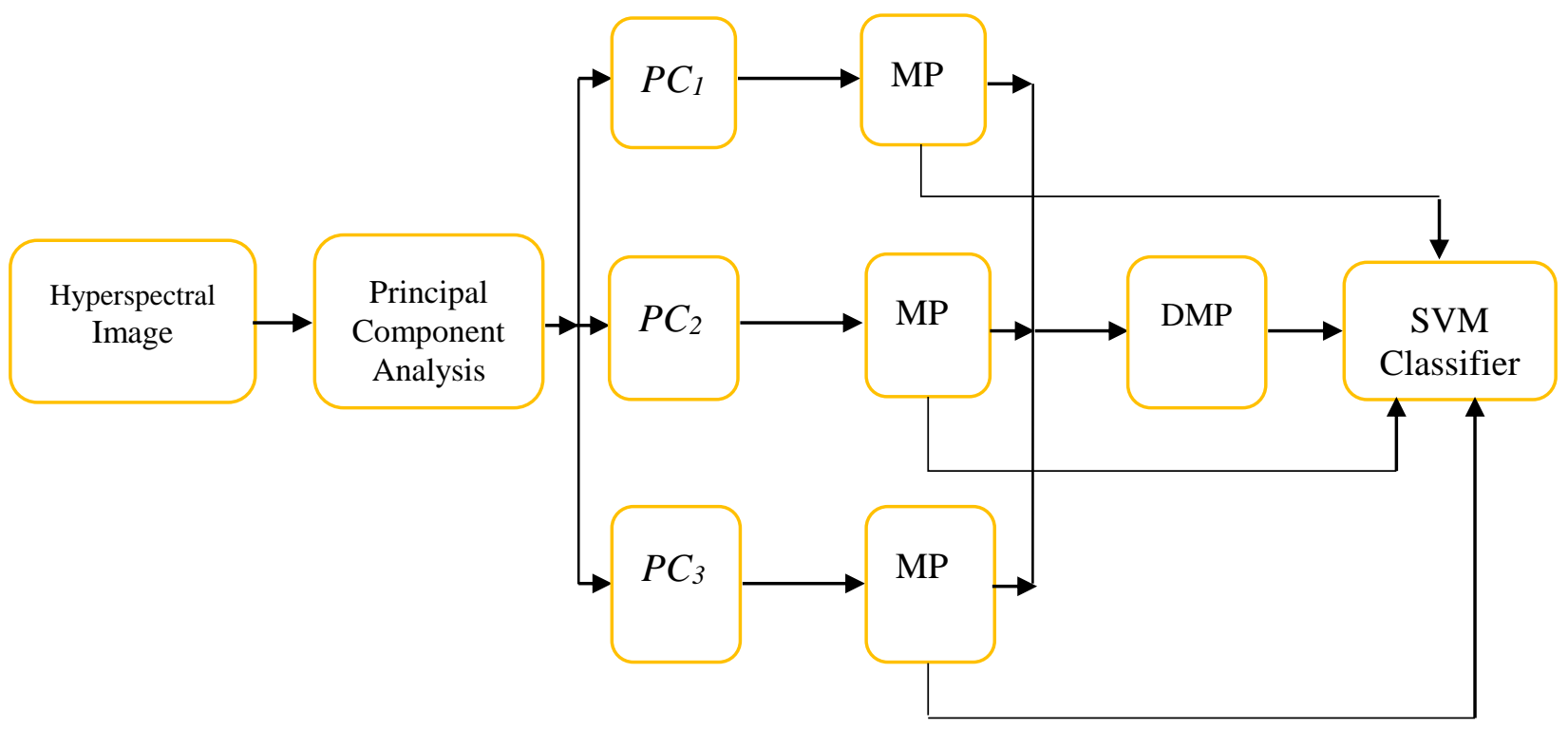

Figure 1. Block diagram of the proposed method for spatial feature extraction.

\subsection{Differential Morphological Profiles}

The differential morphological profiles are obtained by morphological filters. These filters extract important information such as edge and structural form of the targets from the images. In the proposed method, reconstruction-based morphological filters are used to apply this filtering using different structural (circular, linear, rectangular) and different dimensional factors on the image, and then the result of the difference of each dimension and different structural elements are considered as output (Chanussot et al. 2006), (Imani and Ghassemian, 2016c). Differential morphological reconstruction based filters are given on the following equations.

$$
\begin{aligned}
& \Omega_{\omega R}(g)=\left\{\Omega_{\omega i}: \Omega_{\omega i}=\Omega_{R}^{i}(g), \forall i \in[0, \ldots, n]\right\} \\
& \Omega_{\delta R}(g)=\left\{\Omega_{\delta i}: \Omega_{\delta i}=\delta_{R}^{i}(g), \forall i \in[0, \ldots, n]\right\}
\end{aligned}
$$

Where $\Omega_{\omega R}(g)$ means a reconstruction-based opening-opening morphology filter on an image that is filtered and $\mathrm{i}$ is the different used size. And similarly, $\Omega_{\delta R}(g)$ means the closing morphology filter based on the reconstruction (Benediktsson et al. 2003).

\subsection{Proposed Method}

In this section, the proposed method is introduced. For derivation spatial information from the hyperspectral data, at first, the principal component analysis (PCA) is used, we select the components that have the most information. Here, the first 3 bands of the data PCA1, PCA2 and PCA3 represent the transformation of the main component of the data into a coordinate system or independent vector space. This creates a spatial transformation in which data is arranged based on the amount of variance, the data with the most variance is transferred to the first dimension, and so the rest of the data is arranged, so that the last main component has the least variance. This can be interpreted as some of the dimension has a small share in the resolution and can be ignored.
Then, the morphological filters are applied to extract spatial features of $P C A_{1}, P C A_{2}$, and $P C A 3$ individually. In this work, three different structural elements have been used. 1) circularshaped structural element with a radius of (1 to 20 with step size of 1),2) linear structural element with a length of (1 to 10 with step size of 1 and angle of 90,135),3) rectangular structural element with a length of $(4,8)$ and width $(8,20)$. After derivation the structural element of the circular-shaped, the output is given to the classifier. The outputs of linear and rectangular structural elements are given to the classifier directly. In this experiment, we use SVM classifier which is implemented by LIBSVM (Chang and Lin, 2011) to classify data, in this method, the LIBSVM library is used with binomial kernels. The block diagram of the proposed method is shown in Figure 1.

\section{EXPERIMENTS RESULTS}

In this proposed method, two hyperspectral data are used for implementation. The first hyperspectral data taken by satellite ROSIS is related to the University of Pavia, Italy. Data size is $610 \times 340$ with spatial resolution of 1.3 meters per pixel. This data includes 9 different classes and 103 spectral bands. The second hyperspectral data is related to the Pavia Center, Italy. The data is also taken by the ROSIS satellite. Data size is $1096 \times 715$ pixels with spatial resolution of 1.3 meters per pixel, consisting of 9 classes and 102 spectral bands. These datasets are shown in Figures 2 and 3.

Pavia University and Pavia Center data collection are employed to evaluate the proposed approach. The University of Pavia dataset consists of 42,776 and Pavia Center dataset consists of 148,152 hyperspectral data. In this paper, we use 15,25 , and 50 labeled data to train the supervised part of each class. We also use 0.97 energy of pixels from University of Pavia dataset and Pavia Center dataset. So the first three PCs are selected. The classification results for University of Pavia and Pavia Center datasets are represented in Tables 1 and 2 respectively. Each of these tables has 5 columns. The first column is the number of random training samples. These numbers are 50, 25, 15 samples per class. In the second column, only spectral information is 
used to classify the data (spectral information is given directly from the data to the classifier). In the third column of the tables, only the extracted spatial information using the proposed method are included in the classification. In the fourth column, the data are classified using spectral and spatial information (spatial data are extracted directly from morphological filters without the use of differential morphological filters). In the fifth column spatial information of the proposed method and the spectral information are used for classification of the data, which is the best performance of the classifier in this section. Classification maps of the datasets are shown in Figures 4 and Figures 5.
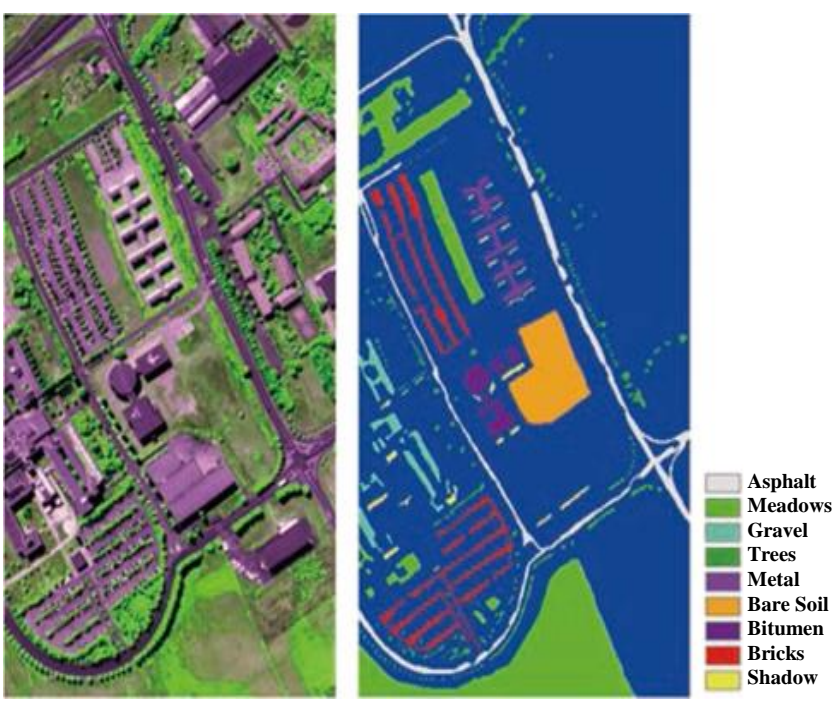

Figure 2. Represents the University of Pavia dataset, Italy, including the RGB map and 9 classes land cover
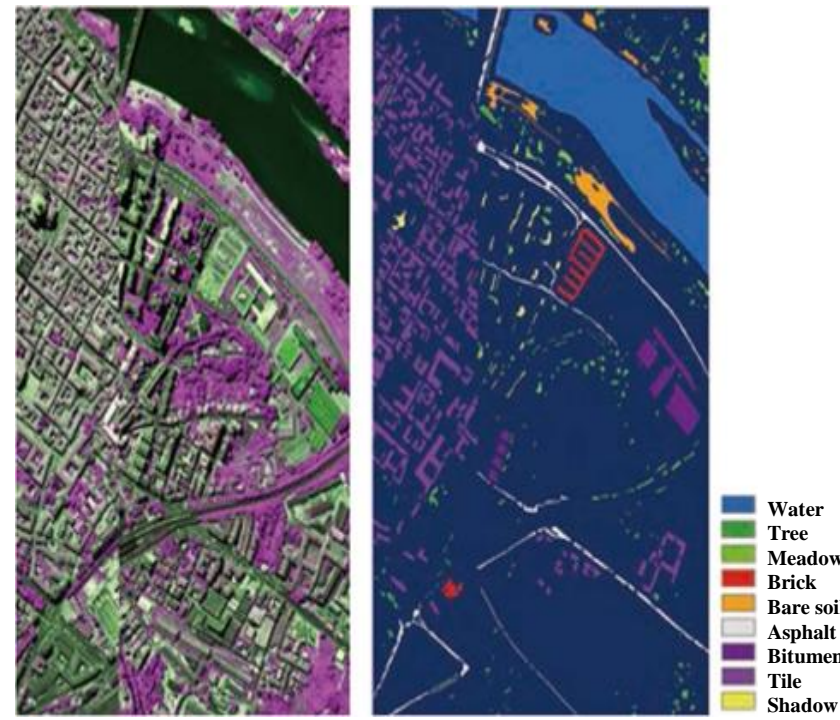

Figure 3. Represents the Pavia Center dataset, Italy, including the RGB map and 9 classes land cover.

Table 1: Overall accuracy (OA) on the Pavia University dataset

\begin{tabular}{|c|c|c|c|c|}
\hline & \multicolumn{4}{|c|}{ SVM Classifier } \\
$\begin{array}{c}\text { Number of } \\
\text { Training } \\
\text { Sample }\end{array}$ & Spectral & $\begin{array}{c}\text { Spatial } \\
\text { propose } \\
\text { d } \\
\text { method }\end{array}$ & $\begin{array}{c}\text { Spatial } \\
\text { MP } \\
+ \text { Spectral }\end{array}$ & $\begin{array}{c}\text { Spatial proposed } \\
\text { method } \\
+ \text { Spectral }\end{array}$ \\
\cline { 2 - 5 } & 83.11 & 94.11 & 93.06 & $\mathbf{9 4 . 8 3}$ \\
\hline 50 & 79.32 & 91.11 & 85.27 & $\mathbf{9 2 . 5 6}$ \\
\hline 15 & 75.57 & 87.27 & 81.17 & $\mathbf{8 6 . 7 2}$ \\
\hline
\end{tabular}

Table 2: Overall accuracy (OA) on the Pavia Center dataset

\begin{tabular}{|c|c|c|c|c|}
\hline \multirow{2}{*}{$\begin{array}{c}\text { Number } \\
\text { of } \\
\begin{array}{c}\text { Training } \\
\text { Sample }\end{array}\end{array}$} & \multicolumn{4}{|c|}{ SVM Classifier } \\
\cline { 2 - 5 } & Spectral & $\begin{array}{c}\text { Spatial } \\
\text { proposed } \\
\text { method }\end{array}$ & $\begin{array}{c}\text { Spatial } \\
\text { MP } \\
+ \text { Spectral }\end{array}$ & $\begin{array}{c}\text { Spatial } \\
\text { proposed } \\
\text { method } \\
+ \text { Spectral }\end{array}$ \\
\hline 50 & 96.67 & 95.25 & 95.89 & $\mathbf{9 6 . 9 1}$ \\
\hline 25 & 95.59 & 93.39 & 94.65 & $\mathbf{9 5 . 4 4}$ \\
\hline 15 & 94.78 & 92.33 & 92.10 & $\mathbf{9 3 . 1 6}$ \\
\hline
\end{tabular}

The classification results for University of Pavia and Pavia center datasets are represented in Tables I and II respectively.

\section{CONCLUSION}

In this paper, the concept of (MP) and (DMP) is used to classify remote sensing hyperspectral images. (MP) is one of the most important tools for extract spatial features. In this paper, we have used three different structural elements combining with the opening filters to classification data.in the proposed method, only the output of the disk-shaped structural element is derived and then given to the classifier and other factors are given directly to the classifier. According to the table I, II for the Pavia University and Pavia center dataset, we can see that with 50 training sample spectral+spatial features overall accuracy using (proposed method) has improved approximately 2\% compared to spectral+spatial features using (MP). In this paper, only opening filters are used to extract spatial features, and the outputs of these filters are given to the inputs of the differential filters, by using this method we consider the output of different images individually for each of the different dimensions and structural elements as outputs. And we consider the different structural elements as outputs, while in other common ways open and closing filters are used and the outputs of these filters are classification. 


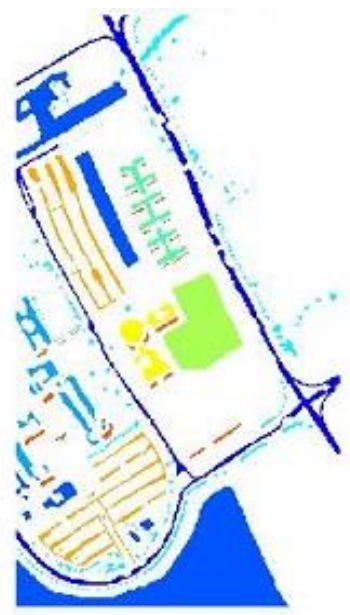

(a)

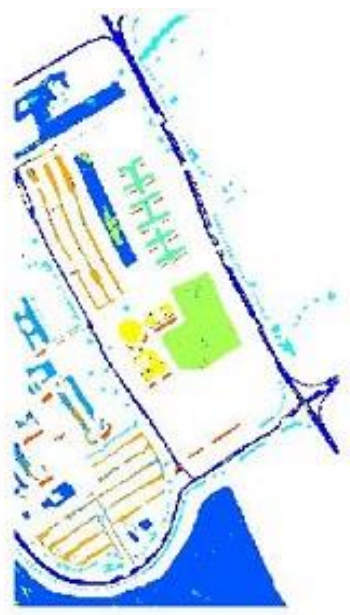

(b)

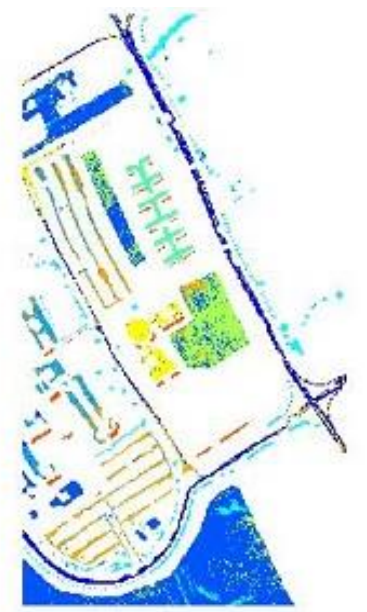

(c)

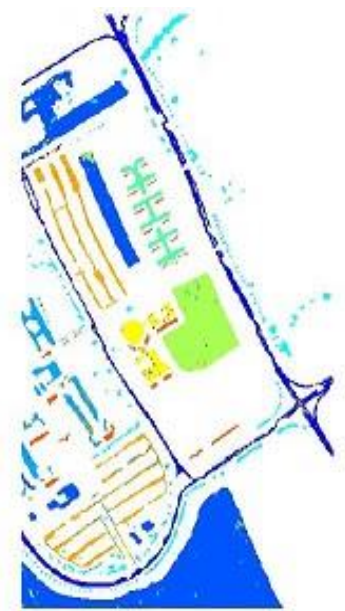

(d)

Figure 4. Classification map for the University of Pavia dataset. (a) Reference map, (b) Spatial proposed method, (c) spectral, (d) spectral-spatial proposed method

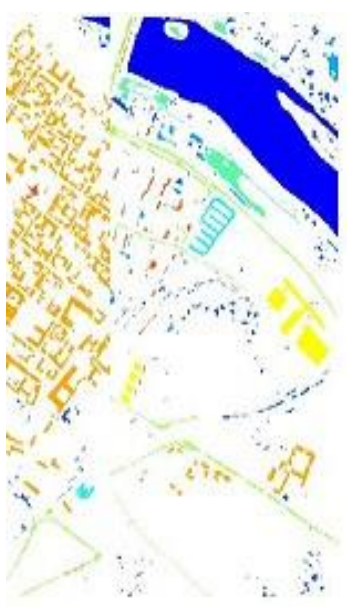

(a)

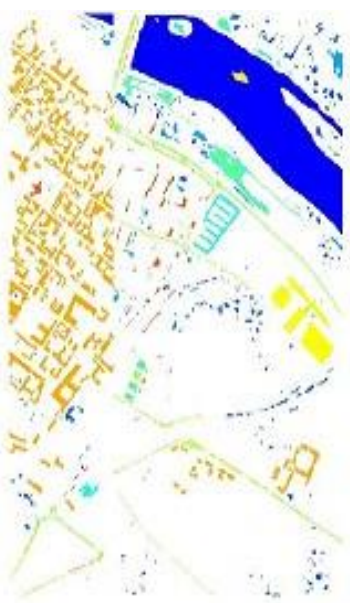

(b)

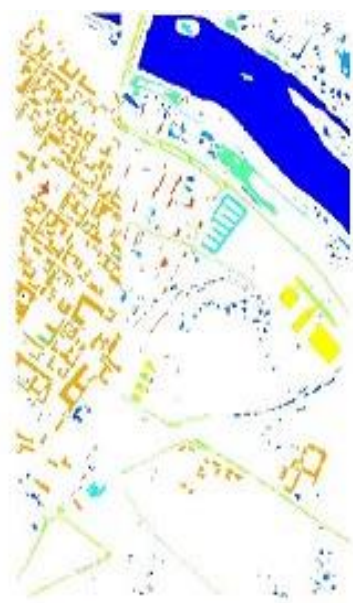

(c)

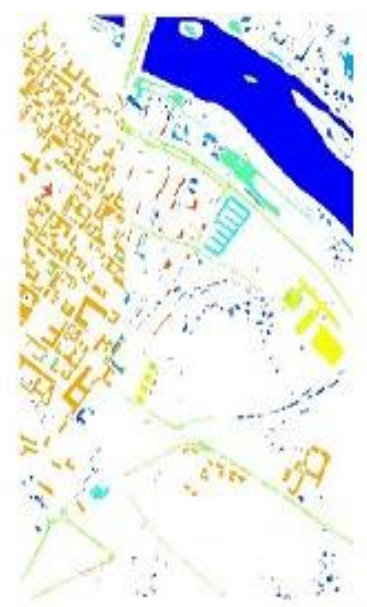

(d)

Figure 5. Classification map for the Pavia center dataset. (a) Reference map. (b) Spectral, (c) Spatial proposed method, (d) spectralspatial proposed method

\section{REFERENCES}

Benediktsson, J.A., Palmason, J.A. and Sveinsson, J.R., 2005. Classification of hyperspectral data from urban areas based on extended morphological profiles. IEEE Transactions on Geoscience and Remote Sensing, 43(3), pp.480-491.

Benediktsson, J.A., Pesaresi, M. and Amason, K., 2003. Classification and feature extraction for remote sensing images from urban areas based on morphological transformations.IEEE Transactions on Geoscience and Remote Sensing, 41(9), pp.1940-1949.
Dalla Mura, M., Atli Benediktsson, J., Waske, B. and Bruzzone, L., 2010a. Extended profiles with morphological attribute filters for the analysis of hyperspectral data, International Journal of Remote Sensing, 31(22), pp.5975-5991.

Chaji, N., Ghassemian, H., 2006. Texture Gradient Based Contour Detection", EURASIP Journal on Applied Signal Processing, 2006 (13), Article ID 21709, pp.1-8. 2006.

Chang, C.C. and Lin, C.J., 2011. LIBSVM: A library for support vector machines. ACM transactions on intelligent systems and technology (TIST), 2(3), p.27 
Chanussot, J., Benediktsson, J.A. and Fauvel, M., 2006. Classification of remote sensing images from urban areas based on morphological transformations. IEEE Transactions on Geoscience and Remote Sensing, 41(9), pp.1940-1949.

Chanussot, J., Benediktsson, J.A. and Fauvel, M., 2006. Classification of remote sensing images from urban areas using a fuzzy possibilistic model. IEEE Geoscience and Remote Sensing Letters, 3(1), pp.40-44.

Dalla Mura, M., Benediktsson, J.A., Waske, B. and Bruzzone, L., 2010b. Morphological attribute profiles for the analysis of very high resolution images. IEEE Transactions on Geoscience and Remote Sensing, 48(10), pp.3747-3762.

Fauvel, M., Chanussot, J., Benediktsson, J.A. and Sveinsson, J.R., 2007, July. Spectral and spatial classification of hyperspectral data using SVMs and morphological profiles. 2007 IEEE International Geoscience and Remote Sensing Symposium (pp. 4834-4837). IEEE Xplore.

Fauvel, M., Tarabalka, Y., Benediktsson, J.A., Chanussot, J. and Tilton, J.C., 2013. Advances in spectral-spatial classification of hyperspectral images. Proceedings of the IEEE, 101(3), pp.652-675.

Ghassemian H., Landgrebe, D., 1988a. On-line object feature extraction for multispectral scene representation, NASA Technical Report Server, TR-EE-88-34. http://ntrs.nasa.gov/search.jsp?R=19900019572 \&qs=Nm\%3D4 $293585563 \% 7$ CAuthor\%7CGhassemian\%2C\%2520Hassan\%26 N\%3D0.

Ghassemian H., Landgrebe, D., 1988b. Object-Oriented Feature Extraction Method for Image Data Compaction, IEEE Control System Magazine, 8 (3),pp. 42-46.

Golipour, M., Ghassemian, H. and Mirzapour, F., 2016. Integrating hierarchical segmentation maps with MRF prior for classification of hyperspectral images in a Bayesian framework. IEEE Transactions on Geoscience and remote Sensing, 54(2), pp.805-816.

Imani, M. and Ghassemian, H., 2016a. Edge patch image-based morphological profiles for classification of multispectral and hyperspectral data. IET Image Processing, 11(3), pp.164-172.

Imani, M. and Ghassemian, H., 2016b, May. GLCM, Gabor, and morphology profiles fusion for hyperspectral image classification. In 2016 24th Iranian Conference on Electrical Engineering (ICEE) (pp. 460-465). IEEE Xplore.

Imani, M. and Ghassemian, H., 2016c, September. Hyperspectral images classification by spectral-spatial processing. In 2016 8th International Symposium on Telecommunications (IST) (pp. 456-461). IEEE Xplore.

Imani, M. and Ghassemian, H., 2017. Edge-preserving-based collaborative representation for spectral-spatial classification. International Journal of Remote Sensing, 38(20), pp.5524-5545.

Khodadadzadeh, M., Ghassemian, H., 2011. Contextual Classification of Hyperspectral Remote Sensing Images Using SVM-PLR, Australian Journal of Basic and Applied Sciences, 5 (8), pp 374-382.
Kianisarkaleh, A. and Ghassemian, H., 2016. Nonparametric feature extraction for classification of hyperspectral images with limited training samples. ISPRS Journal of Photogrammetry and Remote Sensing, 119, pp.64-78.

Mirzapour, F. and Ghassemian, H., 2013. Using GLCM and Gabor filters for classification of PAN images, 21th Iranian conference, Mashhad, Iran; 14 - 16 May 2013. IEEE Xplore.

Mirzapour, F. and Ghassemian, H., 2016a. Moment-based feature extraction from high spatial resolution hyperspectral images. International Journal of Remote Sensing, 37(6), pp.1349-1361.

Mirzapour, F. and Ghassemian, H., 2016b. Multiscale Gaussian derivative functions for hyperspectral image feature extraction. IEEE Geoscience and Remote Sensing Letters, 13(4), pp.525529 .

Pesaresi, M. and Benediktsson, J.A., 2001. A new approach for the morphological segmentation of high-resolution satellite imagery. IEEE transactions on Geoscience and Remote Sensing, 39(2), pp.309-320.

Plaza, A., Martinez, P., Plaza, J. and Perez, R., 2005. Dimensionality reduction and classification of hyperspectral image data using sequences of extended morphological transformations. IEEE Transactions on Geoscience and remote sensing, 43(3), pp.466-479.

Roochi, N.G., Ghassemian, H. and Mirzapour, F., 2017, September. Remote sensing images classification using moment features and attribute profiles. In 2017 IEEE International Conference on Signal and Image Processing Applications (ICSIPA) (pp. 49-54). IEEE Xplore. 